\title{
EFEITOS DA ANSIEDADE SOBRE A PRESSÃO ARTERIAL EM MULHERES COM HIPERTENSÃO ${ }^{1}$
}

Chaves EC, Cade NV. Efeitos da ansiedade sobre a pressão arterial em mulheres com hipertensão. Rev Latino-am Enfermagem 2004 março-abril; 12(2):162-7.

Estudo descritivo, associativo, que objetiva conhecer a relação da ansiedade com os níveis de pressão arterial em mulheres hipertensas e da ansiedade com o tempo de tratamento da hipertensão. Foram pesquisadas 78 mulheres em tratamento para hipertensão no InCor, mediante o Inventário de Ansiedade de Spilberger - IDATE, e a pressão arterial verificada, utilizando-se da medida indireta, obtida pelo método auscultatório. Os dados foram submetidos à análise estatística, com nível de significância de 5\%. A amostra apresentou traço e estado de ansiedade moderados e médias de pressão acima do normal, compatível com hipertensão estágio 1. Não houve diferença estatisticamente significante entre pressão arterial e níveis de ansiedade e entre o tempo de tratamento para hipertensão e níveis de ansiedade.

DESCRITORES: ansiedade; pressão arterial; hipertensão

\section{ANXIETY EFFECTS ON BLOOD PRESSURE OF WOMEN WITH HYPERTENSION}

This descriptive and associative study aims to examine the relation between anxiety and blood pressure levels in women with hypertension and between anxiety and hypertension treatment time. A research was carried out among 78 women under hypertension treatment at InCor Hospital, Brazil, through Spilberger Anxiety Inventory - IDATE and blood pressure was checked by means of the indirect measure, obtained by the auscultatory method. Data were submitted to statistic analysis at a $5 \%$ significance level. The sample displayed moderate traces and states of anxiety and average blood pressure above normal, compatible with hypertension stage 1. No significant statistical difference was observed between blood pressure and anxiety levels, nor between hypertension treatment time and anxiety levels.

DESCRIPTORS: anxiety; blood pressure; hypertension

\section{EFECTOS DE LA ANSIEDAD SOBRE LA PRESIÓN ARTERIAL EN MUJERES CON HIPERTENSIÓN}

Estudio descriptivo, asociativo que tuvo como objetivo conocer la relación entre la ansiedad y los niveles de presión arterial en mujeres con hipertensión y la ansiedad con el tiempo de tratamiento de la hipertensión. Se trabajó con 78 mujeres en tratamiento para hipertensión en el Instituto del Corazón SP. (INCor), mediante el Inventario de Ansiedad de Spilberger IDATE y la presión arterial se midió a través de una medida indirecta obtenida por el método auscultatorio. Los datos fueron sometidos al análisis estadístico con nivel de significáncia de 5\%. La muestra presentó trazo y estado ansiedad moderados y promedio de presión arriba del normal, compatible con hipertensión nivel 1. No existió diferencia estadística significante entre presión arterial con nivel de ansiedad y entre el tiempo de tratamiento para hipertensión con los niveles de ansiedad.

DESCRIPTORES: ansiedad; presión arterial; hipertensión

\footnotetext{
${ }^{1}$ Este trabalho foi construído a partir da tese de doutorado intitulada "O modelo cognitivo-comportamental em grupo e seus efeitos sobre as estratégias de enfrentamento, os estados emocionais e a pressão arterial de mulheres hipertensas"; ${ }^{2}$ Doutor em Psicologia Social, Docente da Escola de Enfermagem da Universidade de São Paulo; ${ }^{3}$ Doutor em Enfermagem, Docente da Universidade Federal do Espírito Santo, e-mail: nagelavc@terra.com.br
} 
INTRODUÇÃO

$\boldsymbol{A}$ ansiedade pode ser definida como um conjunto de manifestações somáticas - aumento da freqüência cardíaca e respiratória, sudorese, tensão muscular, náusea, vazio no estômago, tonteira e manifestações psicológicas - apreensão, alerta, inquietude, hipervigilância, dificuldade de concentração e de conciliação do sono, entre outros ${ }^{(1)}$.

A ansiedade tem função adaptativa, pois mobiliza os recursos individuais para enfrentamento mais adequado, em face às demandas do cotidiano. Nesse sentido, ela é esperada nas interações humanas e funciona como um alerta, eliciando esforço, atenção, prontidão e um investimento maior em situações que, de alguma forma, ameacem o indivíduo.

Também pode ser um sintoma de um quadro patológico, como no hipertireoidismo; sintoma de quadros psiquiátricos (depressão) ou pode ser uma doença nos diferentes tipos de ansiedade patológica, como pânico e fobia ${ }^{(1)}$.

Quanto à sua relação com o estresse descrito por Selye, em 1936, entende-se ser a ansiedade um dos componentes afetivos do processo de estresse, juntamente com outras emoções, como a raiva e o medo, que podem surgir quando as demandas ambientais são percebidas como algo que excede a capacidade de resposta do indivíduo.

Emoções específicas, hiper-reatividade cardiovascular e sua relação causal com a hipertensão arterial têm sido motivo de muitas pesquisas, porém, uma das dificuldades apontadas nos estudos consiste na dúvida sobre as alterações estruturais no organismo do hipertenso serem decorrentes das modificações que a doença proporciona, ou se antecedem e explicam a hipertensão(2).

Episódios pressores repetidos, advindos das relações interpessoais estressantes, têm mostrado relação com o aceleramento da hipertensão, desde que promovam descargas no sistema nervoso central de forma repetida e acumulada em pessoas geneticamente predisponentes ${ }^{(3-5)}$. Como conseqüência dessas pressões elevadas, ocorre, com o tempo e a freqüência dos estímulos, espessamento da camada muscular do vaso e essa hipertrofia vascular torna o vaso hiper-responsivo, com elevação da pressão arterial|(3,6).

Com base na hipótese de que o estado emocional interfere na variabilidade da pressão arterial, pesquisaramse os efeitos da felicidade, da raiva e da ansiedade em pacientes com Hipertensão Arterial Borderline e concluiuse que os três estados emocionais elevam a pressão arterial, encontrando-se forte associação entre a intensidade da ansiedade e a pressão arterial diastólica ${ }^{(7)}$.

Se, por um lado, emoções promovem alterações cardiovasculares, por outro, estudos mostram elevada morbidade psiquiátrica em hipertensos.

Avaliou-se a morbidade psiquiátrica em hipertensos graves em tratamento ambulatorial e foram encontrados distúrbios psiquiátricos em $65,8 \%$ da amostra. O distúrbio mais freqüente foi o do humordepressão, distimia - distribuído em $46,3 \%$ das mulheres e $19,5 \%$ nos homens ${ }^{(8)}$.

Outros autores estudaram a presença de distúrbios psiquiátricos e a qualidade de vida em homens e mulheres, usuários de serviços primários de saúde, com diferentes doenças crônicas (hipertensão, diabetes, artrite e cardiopatia), partindo do pressuposto que as alterações emocionais interferem com a qualidade de vida dos pacientes. Concluíram que as mulheres apresentaram mais desordens mentais (ansiedade, depressão, desordens somáticas e distúrbios alimentares) e mais comorbidade psiquiátrica ${ }^{(9)}$.

Esses dados não são unânimes, mas esse elevado índice de sintomas psiquiátricos em mulheres tem sido justificado pelas alterações biológicas ou hormonais e por fatores sociais, como os papéis desenvolvidos pela mulher na sociedade ${ }^{(9)}$.

O problema da pesquisa constituiu-se em: o estado de ansiedade aumentado promove aumento da pressão arterial em mulheres hipertensas? Optou-se por trabalhar somente com mulheres devido ao fato de elas serem acometidas por ansiedade duas vezes ou mais em relação aos homens ${ }^{(10)} \mathrm{e}$, dessa forma, pensou-se, com esta amostra, obter escores mais elevados de ansiedade.

\section{OBJETIVOS}

A pesquisa tem por objetivos: a) verificar a relação entre ansiedade e os níveis de pressão arterial em mulheres hipertensas e b) relacionar ansiedade com tempo de tratamento da hipertensão arterial.

\section{MATERIAL E MÉTODO}

A amostra constituiu-se de 78 mulheres com 
diagnóstico médico de Hipertensão Arterial Essencial, em tratamento na Unidade de Hipertensão do Instituto do Coração da Faculdade de Medicina da Universidade de São Paulo (USP-InCor).

O projeto da tese de doutorado que originou esse estudo foi aprovado pelo Comitê de Ética em Pesquisa da Escola de Enfermagem da USP-SP e pela Comissão Científica e de Ética do Instituto do Coração.

Constituíram-se os critérios de inclusão da amostra: sexo feminino, idade superior a 21 anos e saber ler. Foram excluídas pacientes com história de transtorno psiquiátrico e uso de medicação que atue diretamente no psiquismo.

Como instrumento, utilizou-se o Inventário de Ansiedade Traço-Estado de Spilberger (IDATE). Constituise em instrumento de auto-avaliação e apresenta como característica principal a mensuração de aspectos inespecíficos que permeiam as situações estressantes como, por exemplo, tensão, nervosismo, irritabilidade, preocupação, apreensão e outros ${ }^{(11)}$.

O IDATE apresenta dois instrumentos independentes - traço de ansiedade e estado de ansiedade. O Inventário Ansiedade-Traço mensura uma tendência de ação baseada na percepção de situações como uma ameaça, o que tende a elevar a ansiedade, enquanto o Inventário Ansiedade-Estado mede um estado emocional temporário e transitório ${ }^{(11)}$.

Cada inventário contém vinte itens que são respondidos em escala com quatro opções. O Inventário Ansiedade-Traço avalia a freqüência e o Ansiedade-Estado avalia a intensidade do humor, e alguns itens das duas escalas apresentam a pontuação invertida antes de serem somados.

De acordo com as pontuações obtidas, considerase ansiedade traço-estado baixa, quando os valores encontram-se entre 20 a 34 pontos; moderada, entre 35 a 49 pontos; elevada, 50 a 64 pontos; e muito elevada, 65 a 80 pontos $^{(12)}$.

Quanto ao procedimento, consultava-se os prontuários das pacientes no InCor, quanto à possibilidade de fazerem parte do estudo. Caso não houvesse condição clínica impeditiva, a pesquisadora conversava com as pacientes, em sala de espera para a consulta médica, dando informações sobre a pesquisa e confirmando os critérios de inclusão e exclusão.

Quando a paciente manifestava interesse e disponibilidade em participar, anotava-se seus dados de identificação na instituição e o endereço, para um posterior contato para marcação da entrevista, verificação da pressão arterial e preenchimento do IDATE.

A entrevista constou de levantamento dos dados sociodemográficos e, nesse momento, foi assinado 0 termo de consentimento informado.

Uma semana após realizadas as entrevistas, foi agendada, com todas as pacientes, uma data para o preenchimento do IDATE. O instrumento foi preenchido em grupo, exceto quando alguma das pacientes não tinha condições de comparecer no dia e horário marcados. A pesquisadora esteve presente em todo o processo e aplicou o instrumento, procurando esclarecer as dúvidas.

A pressão arterial foi verificada previamente à entrevista e ao preenchimento do IDATE. Quanto à técnica, esperava-se cinco a dez minutos com a paciente em repouso e, após esvaziamento da bexiga, eram realizadas duas verificações consecutivas com dois minutos de intervalo. Convencionou-se que as pressões seriam verificadas no membro superior esquerdo e utilizou-se dois tamanhos de manguito - adulto e adulto grande - de acordo com a circunferência de braço. $O$ manguito foi posicionado dois centímetros acima da fossa antecubital com a bolsa centralizada sobre a artéria braquial. Foi utilizado o aparelho automático marca OMRON - HEM 705 CP, que é validado para esse procedimento. O equipamento foi adquirido para a pesquisa, calibrado pela fábrica.

A coleta de dados ocorreu no primeiro semestre de 2000 .

Quanto ao tratamento estatístico, verificou-se a possível dependência entre a pressão arterial sistólica e pressão arterial diastólica com o estado de ansiedade mediante a ANOVA. Foi usado o teste não-paramétrico de Kruskal-Wallis para ver a possível relação entre tempo de tratamento e ansiedade. O nível de significância foi de $5 \%$.

\section{RESULTADO E DISCUSSÃO}

Sobre as características sociodemográficas, observa-se, na Tabela 1, que, das 78 pacientes, 47 delas $(60,26 \%)$ eram não brancas, 54 (69,24\%) com idade entre 44 a 63 anos, 41 pacientes (52,56\%) casadas/amasiadas, $52(66,66 \%)$ tinham o primeiro grau incompleto.

O tempo médio de tratamento para hipertensão arterial foi de quinze anos, mediana de treze anos e desvio padrão de mais ou menos dez anos. 
Tabela 1 - Características sociodemográficas das pacientes

\begin{tabular}{|c|c|c|c|}
\hline $\begin{array}{l}\text { Características } \\
\text { sociodemográficas }\end{array}$ & Especificação & $\mathrm{n}^{\circ}$ & $\%$ \\
\hline Faixa etária & $\begin{array}{l}24 \mid-44 \\
44 \mid-64 \\
64|-| 72\end{array}$ & $\begin{array}{l}08 \\
54 \\
16\end{array}$ & $\begin{array}{l}10,25 \\
69,24 \\
20,51\end{array}$ \\
\hline Raça & $\begin{array}{l}\text { Branca } \\
\text { Não branca } \\
\text { Amarela }\end{array}$ & $\begin{array}{l}30 \\
47 \\
01\end{array}$ & $\begin{array}{c}38,46 \\
60,26 \\
1,28\end{array}$ \\
\hline Escolaridade & $\begin{array}{l}\text { Sabe ler e escrever } \\
1^{\circ} \text { grau incompleto } \\
1^{\circ} \text { grau completo } \\
2^{\circ} \text { grau incompleto } \\
2^{\circ} \text { grau completo } \\
3^{\circ} \text { grau incompleto }\end{array}$ & $\begin{array}{l}03 \\
52 \\
09 \\
06 \\
07 \\
01\end{array}$ & $\begin{array}{c}3,84 \\
66,66 \\
11,53 \\
7,79 \\
8,97 \\
1,28\end{array}$ \\
\hline Estado civil & $\begin{array}{l}\text { Solteira } \\
\text { Casada/amasiada } \\
\text { Viúva } \\
\text { Separada }\end{array}$ & $\begin{array}{l}10 \\
41 \\
14 \\
13\end{array}$ & $\begin{array}{l}12,82 \\
52,56 \\
17,95 \\
16,67\end{array}$ \\
\hline $\begin{array}{l}\text { Renda per capita } \\
\text { em salário-mínimo }\end{array}$ & $\begin{array}{l}\text { Sem renda } \\
<1 \mathrm{SM} \\
1 \mid-4 \\
4|-| 8 \\
>8 \mathrm{SM} \\
\text { Total }\end{array}$ & $\begin{array}{l}02 \\
18 \\
47 \\
09 \\
02 \\
78\end{array}$ & $\begin{array}{c}2,56 \\
23,08 \\
60,26 \\
11,52 \\
2,56 \\
100\end{array}$ \\
\hline
\end{tabular}

Na Tabela 2, encontram-se as medidas descritivas das variáveis: ansiedade-traço, ansiedade-estado e pressão arterial sistólica (PAS) e diastólica (PAD). Considerou-se como valores de pressão arterial (PA) a média dos valores da PA verificada nas duas semanas do estudo.

De acordo com a classificação clínica dos resultados obtidos, as pacientes apresentaram média na ansiedade-traço e ansiedade-estado compatível com moderada, o que é considerado como parâmetro dentro da normalidade.

No que diz respeito à média da PAS e PAD, observa-se que os valores estavam acima do normal (normal: <130 x 85mmHg) e compatíveis com hipertensão estágio I (PAS 140-159mmHg ou PAD 90-99mmHg) ${ }^{(13)}$.

Quanto à estatística inferencial, a associação entre pressão arterial sistólica ( $p: 0,301$ ), pressão arterial diastólica ( $p: 0,356)$ com ansiedade-estado não se mostrou significativa (Tabela 3).

Tabela 2 - Medidas descritivas das variáveis: ansiedadetraço, ansiedade-estado, pressão arterial sistólica e diastólica

\begin{tabular}{lcccc}
\hline \multicolumn{1}{c}{ Variáveis } & $\begin{array}{c}\text { Valor } \\
\text { Mínimo }\end{array}$ & $\begin{array}{c}\text { Valor } \\
\text { Máximo }\end{array}$ & Média & $\begin{array}{c}\text { Desvio } \\
\text { Padrão }\end{array}$ \\
\hline Ansiedade-traço & 23 & 71 & 47 & \pm 12 \\
Ansiedade-estado & 20 & 66 & 39 & \pm 12 \\
Pressão arterial sistólica & 105 & 220 & 150 & \pm 28 \\
Pressão arterial diastólica & 66 & 131 & 89 & \pm 13 \\
\hline
\end{tabular}

Tabela 3 - Distribuição das pacientes de acordo com o nível de ansiedade, segundo a média da pressão arterial sistólica e diastólica

\begin{tabular}{lcccc}
\hline $\begin{array}{l}\text { Classificação da } \\
\text { ansiedade-estado }\end{array}$ & $\mathrm{n}$ & $\%$ & $\begin{array}{c}\text { Média } \\
\text { PAS }\end{array}$ & $\begin{array}{c}\text { Média } \\
\text { PAD }\end{array}$ \\
\hline Baixa & 36 & 46,15 & 144 & 87 \\
Moderada & 28 & 35,90 & 142 & 86 \\
Elevada & 11 & 14,10 & 132 & 82 \\
Muito elevada & 03 & 3,85 & 160 & 97 \\
Total & 78 & 100 & & \\
\hline
\end{tabular}

Quais os possíveis motivos de a ansiedade não mostrar associação com a pressão arterial neste estudo? Serão especuladas algumas hipóteses que permeiam essa temática.

De acordo com alguns autores ${ }^{(7)}$, haveria necessidade de intensa ansiedade para elevar a pressão arterial e, no presente estudo, foi pequeno o número de pacientes com ansiedade elevada e muito elevada - 14 $(17,95 \%)$ sujeitos.

Observa-se, na Tabela 3, que somente três mulheres apresentavam ansiedade "muito elevada" e, nesse nível de ansiedade, as médias da pressão sistólica e da diastólica estavam mais elevadas do que as demais. Mas, quando a ansiedade estava somente "elevada" (14,10\% das mulheres), a média caiu.

Pesquisadores ${ }^{(14)}$, ao estudarem em população geral a possível relação entre o traço e estado de ansiedade e a reatividade cardiovascular ao estresse eliciado em laboratório, mediante testes que estimulam o sistema cardiovascular de maneira distinta - o isométrico e o mental -, encontraram que indivíduos com ansiedade mais elevada apresentaram pressões mais baixas e viceversa. Como os dois testes aplicados envolvem receptores beta adrenérgicos e é sabido que o aumento do tono autonômico promove baixa regulação desses receptores, os autores tentam explicar os resultados do estudo, sugerindo que o aumento da resposta simpática seria uma resposta inicial da ansiedade elevada que, ao tornar-se crônica, promoveria uma baixa regulação desses receptores como forma compensatória. Desse modo, a cronificação da ansiedade levaria a mudanças na reatividade cardiovascular, justificando os resultados do presente estudo.

A ausência de elevação da ansiedade observada no presente estudo poderia indicar que as pacientes estavam aparentemente adaptadas às problemáticas do 
cotidiano, com a maneira que escolheram para enfrentálas, sejam elas conscientes, como as estratégias de enfrentamento, ou inconscientes, como os mecanismos de defesa.

A ansiedade avaliada neste trabalho refere-se à ansiedade supostamente natural, como fenômeno da vida cotidiana, necessária ao ajuste dos recursos da pessoa à situação vivenciada e conseqüente adaptação do indivíduo ao meio. Nesse caso, a ansiedade eleva-se diante da possibilidade de os projetos de vida e as expectativas não irem adiante ou estarem ameaçados de se concretizarem, ameaça essa que ocorre de acordo com a trama de significados simbólicos preexistentes.

A partir dessa premissa, infere-se que as pacientes não estavam vivenciando uma situação ansiogênica no momento da coleta de dados. Possivelmente, mais ansiedade poderia ser verificada, caso as pacientes estivessem sob um evento de vida estressante, que demandasse mais esforço por parte delas.

Essa asserção é corroborada por resultados de outros estudos. Pacientes a serem submetidos a cirurgia cardíaca que apresentavam ansiedade, mostraram aumento da reatividade pressórica, mesmo não sendo hipertensos ${ }^{(15)}$.

Outra forma de se compreender os resultados seria pensar que a ansiedade não é uma emoção capaz de elevar a pressão arterial em hipertensas ou que existam outros aspectos envolvidos nesse processo. Estudo mostra que a supressão da expressão da raiva é mais relevante do que a ansiedade, no que tange ao aumento da pressão arterial por mecanismos emocionais ${ }^{(16)}$.

Um dos motivos para que o estado de ansiedade não promova aumento da pressão arterial poderia ser explicado pelo uso de mecanismos de defesa diante das situações ansiogênicas e à alexitimia* Autores afirmam que, dentre as diferentes emoções surgidas diante de um estímulo, podem estar presentes a repressão das emoções e a alexitimia ${ }^{(16)}$. Na repressão das emoções, ocorre o uso de mecanismos de defesa, inibindo assim a ansiedade e, na alexitimia, ocorre baixa expressão dos sentimentos de tal forma que a ansiedade não fique evidente.

Continuando a discorrer sobre o significado dos resultados, na presença de estímulos ansiogênicos, mais de um sistema é ativado, podendo acionar diferentes respostas fisiológicas em um mesmo momento, o que torna difícil diferenciar o que está prevalecendo em dada situação $^{(16)}$.

Constata-se haver dois grandes padrões de respostas emitidas diante da dinâmica da vida. Há aquelas permeadas por ansiedade, as quais geram atitudes de luta, combate, confrontação, consideradas como comportamento ativo, e há aquelas respostas de retirada, de perda de controle e sensação de derrota, com predomínio de sentimentos de tristeza ansiosa ou depressão, denominados de comportamentos passivos ${ }^{(16)}$.

Esses diferentes comportamentos ocorrem devido ao fato de o processo cognitivo proporcionar a liberação de neurotransmissores sinápticos (serotonina, GABA e outros), excitando ou inibindo os neurônios. Esses, por sua vez, modificam a atividade dos diferentes componentes cerebrais e, como resposta a esse padrão hormonal, surgem comportamentos e afetos correspondentes a ele $^{(16)}$.

Nesse sentido, de acordo com a resposta neuroendócrina, a pessoa sente-se desafiada e motivada a se esforçar para combater a situação, com algum nível de controle, ou apresenta sensação de perda de controle, derrota, frustração, com comportamento de imobilismo, desesperança e estado depressivo ${ }^{(16)}$.

As tentativas freqüentes em defender-se e posicionar-se frente aos eventos cotidianos e suas dificuldades, ao gerar frustração e insucessos, pessoais ou sociais, conduzem à mudança de um padrão de comportamento "ativo" para "passivo deprimido"(16).

No presente estudo foi abservado maior contingente de mulheres com ansiedade baixa e moderada e entendeu-se que as condições socioeconômicas menos privilegiadas e suas conseqüências na hierarquia social sejam um dos dificultadores de enfrentamento ativo com maior possibilidade de advirem insucessos, devido à precariedade dos recursos, internos e externos, e falta de perspectiva na escalada social.

Nesta amostra, houve predominância de mulheres acima de cinqüenta anos, com renda per capita um pouco maior que dois salários-mínimos, em sua maioria, sem história de trabalho fora da esfera doméstica, escolaridade

\footnotetext{
* Aleximia pode ser compreendida como “...inabilidade em experienciar, reconhecer e verbalizar sentimentos”. Há diminuição da sensibilidade afetiva, impedindo que o indivíduo perceba e avalie seus sintomas ${ }^{(16)}$
} 
baixa, até o primário, um terço delas não percebem rede de apoio, e 50\% apresentam outras doenças, além da hipertensão, iniciada há mais de dez anos. Supostamente, apresentam repertório comportamental menos desenvolvido pelas condições de vida e menores possibilidades e expectativas diante dos problemas.

Justifica-se, com essas características, a dificuldade em agir ativamente como resposta aos estímulos e, possivelmente, preferindo estilos defensivos, permeados de passividade e comportamento de retirada. Nesse sentido, o estado depressivo poderia caracterizar mais essa amostra, em vez da ansiedade.

Considerou-se, ainda, neste estudo, que o tempo de tratamento para a hipertensão arterial poderia promover maior adaptação da hipertensa aos estímulos ambientais, no que diz respeito às situações ansiogênicas.

Essa hipótese não foi confirmada, e a análise inferencial não se mostrou significativa, pois a ansiedadetraço $(p: 0,666)$ e ansiedade-estado $(p: 0,222)$ não apresentaram associação com o tempo de tratamento (Tabela 4).

\section{REFERÊNCIA BIBLIOGRÁFICA}

1. Dratcu L, Lader M. Ansiedade: conceito, classificação e biologia. J Bras Psiq 1993; 42(1):19-32.

2. Sherwood A, Hinderliter AL, Light KC. Psysiological determinants of hyperreactivity to stress in bordeline hypertension. Hypertension 1995; 25(3):384-90.

3. Julius S, Amerena J, Smith S, Petrin J. Autonomic nervous and behavioral factors in hypertension. In: Laragh $\mathrm{JH}$, Brenner BNM. Hypertension: pathophysiology, diagnosis and management. New York: Raven Press; 1995. p. 2557-68.

4. Faye K, Heng LH, Collomp R, Pereoux E. Hypertension and stress. J Mal Vasc 2003; 28(1):4-8.

5 . Kaplan MS. The psychosocial determinants of hypertension. Nutr Metab Cardiovasc Dis 2003; 13(1):52-9.

6. Folkow B. Cardiovascular structural adaptations: its role in the initiation and maintenance of primary hypertension. Clin Sci Mol Med 1978; 55:3s-22s.

7. James GD, Yee LS, Harshfield GA, Blank SG, Pickering TG. The influence of happiness, anger, and anxiety on the blood pressure of bordeline hypertensives. Psycosom Med 1986; 48(7):502-8.

8. Benseñor IM, Pereira AC, Tannuri AC, Valeri CM, Akashi D, Fucciolo DQ et al. Hipertensão arterial sistêmica e morbidade psiquiátrica em ambulatório de hospital terciário. Arq Neuropsiquiatria 1998; 56(3-A):406-11.

9. Linzer M, Wisconsin M, Spitzer R, Kroenke K, Maryland B, Williams JB et al. Gender, quality of life, and mental disorders in primary care: results from the PRIME-MD 1000 study. Am J Med 1996; 101(5):526-33.
Tabela 4 - Distribuição da classificação ansiedade-estado, segundo o tempo de tratamento em anos

\begin{tabular}{lccc}
\hline Classificação da ansiedade-estado & \multicolumn{3}{c}{ Tempo de Tratamento } \\
& $\mathrm{n}$ & $\%$ & Média \\
\hline Baixa & 36 & 46,15 & 17 \\
Moderada & 28 & 35,90 & 12 \\
Elevada & 11 & 14,10 & 14 \\
Muito elevada & 03 & 3,85 & 8 \\
Total & 78 & 100 & \\
\hline
\end{tabular}

\section{CONCLUSÃO}

Não foi encontrada diferença estatisticamente significante entre pressão arterial sistólica, pressão arterial diastólica e os níveis de ansiedade, em nível de significância de $5 \%$, e nem entre o tempo de tratamento para hipertensão arterial e os níveis de ansiedade nas mesmas condições.

Sugere-se repetição deste estudo com amostra mais representativa, quanto à ansiedade elevada e muito elevada.

10. Clark DM. Estados de ansiedade: pânico e ansiedade generalizada. In: Hawton K, Salkovskis PM, Kirk J, Clark DM. Terapia cognitivo-comportamental para problemas psiquiátricos: um guia prático. São Paulo (SP): Martins Fontes; 1997. p. 75-137.

11. Andrade LHSG, Gorenstein C. Aspectos gerais das escalas de avaliação de ansiedade. Psiq Clin 1998; 25(6 ed. esp.):285-90.

12. Dela Coleta JA, Dela Coleta MF. Escalas para medida de atitudes e outras variáveis psicossociais. Ribeirão Preto (SP): Escola de Enfermagem de Ribeirão Preto/USP; 1996.

13. Sociedade Brasileira de Hipertensão. $3^{\circ}$ Consenso Brasileiro de Hipertensão Arterial. Campos do Jordão (SP); 1998.

14. Young EA, Nesse RM, Weder A, Julius S. Anxiety and cardiovascular reactivity in the Tecumseh population. J Hypertens 1998; 16(12):1727-33.

15. Hallas CN, Thornton EW, Fabri BM, Fox MA, Jackson M. Prediting blood pressure reactivity and heart hate variability in mood state following coronary artery bypass surgery. Int $\mathrm{J}$ Psychophysiol 2003; 47(1):43-55.

16. Henry J, Grimm CE. Psychosocial mechanisms of primary hypertension. J Hypertens 1990; 8:783-93. 